\title{
Contactless Control of Local Surface Buckling in Photoaligned Gold/ Liquid Crystal Polymer Bilayers
}

\author{
Laurens T. de Haan, ${ }^{\dagger}$ Timo J. J. Willigers, ${ }^{+}$Levina E. A. Wijkhuijs, ${ }^{\ddagger}$ Matthew Hendrikx, \\ Cuong Thai Nguyen, ${ }^{\S}$ Philippe Leclère, ${ }^{\S}$ Anne E. J. Souren, ${ }^{\ddagger}$ Guofu Zhou, ${ }^{\dagger}, \|_{\odot}$ \\ and Michael G. Debije**;
}

${ }^{\dagger}$ SCNU-TUE Joint Lab of Device Integrated Responsive Materials, National Center for International Research on Green Optoelectronics, South China Normal University, Guangzhou 510006, People's Republic of China

${ }^{\ddagger}$ Stimuli-Responsive Functional Materials and Devices, Chemical Engineering and Chemistry, Eindhoven University of Technology, 5600 MB Eindhoven, The Netherlands

${ }^{\S}$ Laboratory for Chemistry of Novel Materials, Department of Chemistry, University of Mons, B 7000 Mons, Belgium

"Shenzhen Guohua Optoelectronics Technology Company Limited, Shenzhen 518110, People's Republic of China

\section{Supporting Information}

\begin{abstract}
Wrinkling is a powerful technique for the preparation of surface structures over large areas, but it is difficult to simultaneously control the direction, period, and amplitude of the wrinkles without resorting to complicated procedures. In this work, we demonstrate a wrinkling system consisting of a liquid crystal polymer network and a thin layer of gold, in which the direction of the wrinkles is controlled by the alignment of the liquid crystal molecules and the average amplitude and period are controlled by a highintensity UV irradiation. The UV exposure represses the amplitude and period dictated by the total exposure. Using photoalignment and photomasks, we demonstrate an unprecedented control over the wrinkling parameters and were able to generate some striking optical patterns. The mechanism of the wrinkle suppression was investigated and appears to involve localized photodegradation at the polymer-gold interface, possibly due to the formation of mechanoradicals.
\end{abstract}

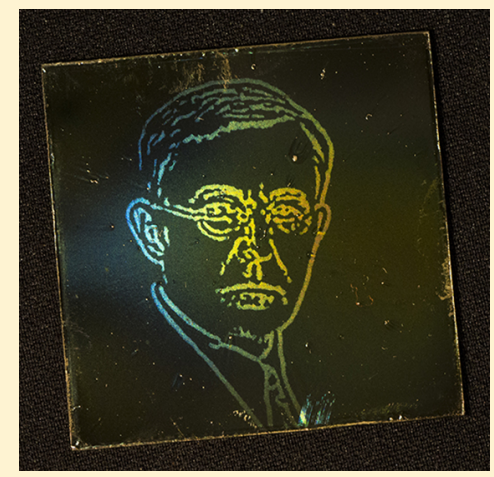

\section{INTRODUCTION}

Controlled formation of structured surfaces is an important subject in materials science. It can give rise to a multitude of fascinating and useful properties in materials, both in nature and in artificial systems, ${ }^{1-3}$ and as such contactless control of surfaces has been a topic of recent research. ${ }^{4-6}$ Surface wrinkling is a powerful way to create structured surfaces over large areas, as the microscopic features are spontaneously generated by a single, macroscopic force without requiring expensive precision equipment, in contrast to other surface modification techniques including printing, laser writing, and lithography. Many interesting applications have been investigated for such patterned surfaces, ${ }^{7}$ including microlens arrays, ${ }^{8}$ antifouling surfaces, ${ }^{9}$ aligned cell growth, ${ }^{10,11}$ phase gratings, ${ }^{12}$ and superhydrophobic surfaces, ${ }^{13}$ among others. A common technique to achieve wrinkling is by subjecting a bilayer, consisting of a thin hard layer on top of a thick soft layer, to a compressive stress. If this stress is higher than a critical stress, determined by the difference in moduli of the hard and soft layers, the strain mismatch between the two layers results in buckling of the thin, hard layer and wrinkles are formed that run perpendicular to the direction of the compression. ${ }^{14}$ Different kinds of material combinations have been investigated, including polystyrene on a liquid crystal (LC) elastomer ${ }^{15,16}$ or on poly(dimethylsiloxane)
(PDMS), ${ }^{12,17-19}$ as well as metals on PDMS, ${ }^{20-23}$ poly(ethylene terephthalate) (PET), ${ }^{24}$ polystyrene, ${ }^{25,26}$ an acrylic elastomer, ${ }^{27}$ and a shape-memory polymer. ${ }^{11,28}$ It is also possible to produce a bilayer system by hardening the surface of a soft layer, for example, with plasma oxidation ${ }^{29-32}$ or by UV exposure. ${ }^{33-35}$

One of the remaining challenges of surface wrinkling is to simultaneously control the direction, amplitude, and period of the wrinkles in a simple way. Many different techniques have been developed to accomplish localized control over the wrinkle direction, such as using patterned relief structures in the soft layer, ${ }^{20,29,33,36}$ using a hard substrate with patterned adhesive properties, ${ }^{37}$ and guiding the wrinkles with an external object, ${ }^{24,38}$ while other techniques have been used to locally affect the wrinkle dimensions, such as using UVozone treatment to locally stiffen PDMS, ${ }^{8,9,23,31}$ direct laser writing, ${ }^{25}$ patterned transfer of the hard layer to the soft layer, ${ }^{19,18,39}$ and preventing the buckling with nanoparticles. ${ }^{40}$ However, all of these techniques are limited to a certain range of possible directional patterns and/or require a lithography step or localized surface modification, which undermines the

Received: June 8, 2018

Revised: $\quad$ August 7, 2018

Published: August 8, 2018 
main strength of surface wrinkling. A technique to fully control both the direction and dimensions of the wrinkles using only simple, low-cost procedures has not yet been reported.

When the soft layer is stretched during the application of the hard layer, subsequent release of this stress can serve as the compressive force that induces wrinkle formation. $., 12,20,22,23,27,29,30,32,35$ In some cases, when a thin metal layer is deposited on a thick polymer layer at a temperature below the polymer's glass transition temperature, a compressive pre-stress is induced by the deposition process, ${ }^{41,42}$ but no wrinkling occurs due to the high modulus of the polymer layer. When the polymer layer is then softened, for example, by application of a solvent, ${ }^{43}$ the critical stress is lowered and the compressive stress can be released by buckling of the hard layer. Earlier, we have shown that, when the thick layer is a photoaligned LC polymer network and the thin layer is a gold film which is applied by sputter coating, wrinkles will form irreversibly upon heating the system beyond the glass transition temperature of the polymer layer, and these wrinkles will follow the alignment of the LC network due to the anisotropic nature of the modulus. ${ }^{44}$ This allows for minute control over the wrinkle direction over arbitrarily large areas, and complex wrinkle patterns can be designed and created. However, while this technique has few limitations when it comes to wrinkle direction, the wrinkle wavelength and amplitude could not be controlled up to this point.

In the work presented here, a new procedure is demonstrated that allows on-demand, local suppression of wrinkle formation in an LC polymer/gold bilayer system through selective application of UV light. An LC polymer network was prepared by spin coating an LC diacrylate solution onto a glass substrate bearing an alignment layer, followed by a low-intensity UV exposure to create an aligned LC polymer network film, to which a gold layer was then applied using sputter coating. Then, high-intensity UV irradiation was locally applied. Finally, the resulting bilayer was heated past the glass transition temperature of the polymer network to induce wrinkle formation. The areas subjected to the high-intensity UV treatment had a visibly reduced wrinkle amplitude and wavelength. This procedure adds a new level of control to the formation of these surface wrinkle patterns, combining for the first time full control of the wrinkle direction with control over the average amplitude and wavelength of the wrinkles using only simple photomasking steps.

\section{EXPERIMENTAL SECTION}

To prepare a homogeneous alignment layer, a 5 wt \% poly(vinyl alcohol) (PVA) solution was applied on cleaned glass plates $(5 \times 5$ $\mathrm{cm}^{2}$ ) by spin coating at $1000 \mathrm{rpm}$ for $30 \mathrm{~s}$ (Karl Suss RC8) and put on an $85{ }^{\circ} \mathrm{C}$ hot plate for $15 \mathrm{~min}$ to evaporate the water. The plates were rubbed on a velvet cloth to form the alignment substrate for the LCs. A different alignment procedure was employed for the photoaligned films: PAAD22 solution (BEAM Company) was diluted $3 \times$ in dimethylformamide (DMF) prior to spin coating on the plates and placed on a $100{ }^{\circ} \mathrm{C}$ hot plate for $10 \mathrm{~min}$ to evaporate the solvent. The PAAD22 photoalignment material was then aligned with polarized UV light $\left(6 \mathrm{~mW} / \mathrm{cm}^{2}\right)$ in two sequential masked steps.

A mixture of the diacrylate liquid crystal LC-242 (BASF), 1 wt \% of the photoinitiator Irgacure 184 (Ciba), and 1 wt $\%$ of the surfactant $\mathrm{N}$-(ethylperfluorooctanesulfamido)ethyl methacrylate (ABCR) was made and dissolved in a 45:55 wt ratio in xylene. The LC mixture was applied to the glass plates bearing alignment layers and spun at 1000 $\mathrm{rpm}$ for $30 \mathrm{~s}$. Immediately after spinning, each substrate was transferred to an $85{ }^{\circ} \mathrm{C}$ hot plate for $15 \mathrm{~s}$ to evaporate the xylene and form the LC nematic phase. After evaporation, the substrate was placed under a low-intensity $\left(<1 \mathrm{~mW} / \mathrm{cm}^{2}\right.$ UVA light $)$ UV lamp (Philips home solaria) under nitrogen flow for $15 \mathrm{~min}$ to photopolymerize the LC mixture. The LC alignment was verified by visual inspection under crossed polarizers. Polymerization was confirmed with Fourier transform infrared spectroscopy (FT-IR) (Varian 670 IR FT-IR spectrophotometer) by attenuated total reflectance (ATR) (Varian 610 IR FT-IR microscope with a germanium crystal). The LC-coated substrates were placed in a sputter coater (Turbo sputter coater K575X dual, Quorum Technologies) and coated with gold at $65 \mathrm{~mA}$ for $60 \mathrm{~s}$, resulting in layers approximately $20 \mathrm{~nm}$ in thickness.

The samples were then partially exposed through the rear (glass) surface by a high-intensity UV lamp (OmniCure series 2000) at various intensities of UVA light. After exposure, the samples were placed on a hot plate at around $75{ }^{\circ} \mathrm{C}$, above the glass transition temperature of the films, to induce wrinkling. To make the samples used for wrinkling parameter measurements, various aligned LC samples were irradiated at different intensities. One-half of the film was covered by an opaque mask to prevent exposure, so the exposed and unexposed parameters could be compared. To generate an image of two elephants, a mask was made by printing the image on paper from a Microsoft Word file, cutting it out, and sputter coating the mask with gold for $15 \mathrm{~s}$ to decrease the permeability to UV. Masked exposure was performed by putting a photoaligned bilayer on a cork ring with the gold side facing down, placing the mask on top with the uncoated side facing up, and placing a glass slide on top to keep the mask flat, followed by irradiation at $26.6 \mathrm{~mW} / \mathrm{cm}^{2}$ for $600 \mathrm{~s}$.

The wrinkling was then measured with a DektakXT profilometer (Bruker). Each sample was measured over a $1 \mathrm{~mm}$ distance in $30 \mathrm{~s}$. The data was processed by first identifying the minima and maxima of the wrinkling. Then, these data points were expanded to match the original number of data points and averaged. The resulting averages were subtracted from the original data, and the resulting corrected profile was used to find the average amplitude and period of the wrinkles.

The modulus of the bulk liquid crystal network (LCN) films was measured with dynamic mechanical thermal analysis (DMTA) (DMA Q800, TA Instruments). The sample was clamped in position, and a torque of $70 \mathrm{cNm}$ was applied. The length of the sample $(5-10 \mathrm{~mm})$ was measured by adding a preload force of $0.01 \mathrm{~N}$. The sample was cooled to and maintained at $0{ }^{\circ} \mathrm{C}$ for $10 \mathrm{~min}$. Thereafter, the sample was heated to $130{ }^{\circ} \mathrm{C}$ at a heating rate of $3{ }^{\circ} \mathrm{C} / \mathrm{min}$. The data were evaluated using TA universal analysis.

To determine the surface modulus at the nanoscale and try to estimate the potential local heterogeneities, intermodulation atomic force microscopy (ImAFM) was used. ${ }^{45}$ In ImAFM the cantilever is driven at two frequencies close to the resonance frequency; the nonlinear character of the tip-surface interaction leads to frequency mixing (intermodulation products). This multifrequency response spectrum can be directly transformed to show how the elastic (conservative) and viscous (dissipative) forces between the tip and surface depend on the amplitude of cantilever oscillation. The information contained in the intermodulation spectrum is therefore sufficient to be able to reconstruct the force-distance curves from which, among the different mechanical parameters, the modulus could be extracted from each pixel of the ImAFM image. The sample was analyzed with a Dimension Icon AFM (Bruker Corporation) fitted with an external multifrequency lock-in amplifier and associated software for calibration and nanomechanical analysis (Intermodulation Products $\mathrm{AB}$ ). ImAFM was done using RTESPA-300 silicon cantilevers (RTESPA-300, $k=40 \mathrm{~N} / \mathrm{m}$, from Bruker Corp.). The resolution of all scans was $512 \times 512$ pixels taken at $0.5 \mathrm{~Hz}$ line rate. The force curves were fitted by using modified DMT modulus.

\section{RESULTS AND DISCUSSION}

The LC polymer films were prepared by spin coating a mixture of the mesogenic monomer with $1 \%$ photoinitiator onto a glass plate which carried a PVA alignment layer to induce monodomain alignment. The monomer was polymerized 


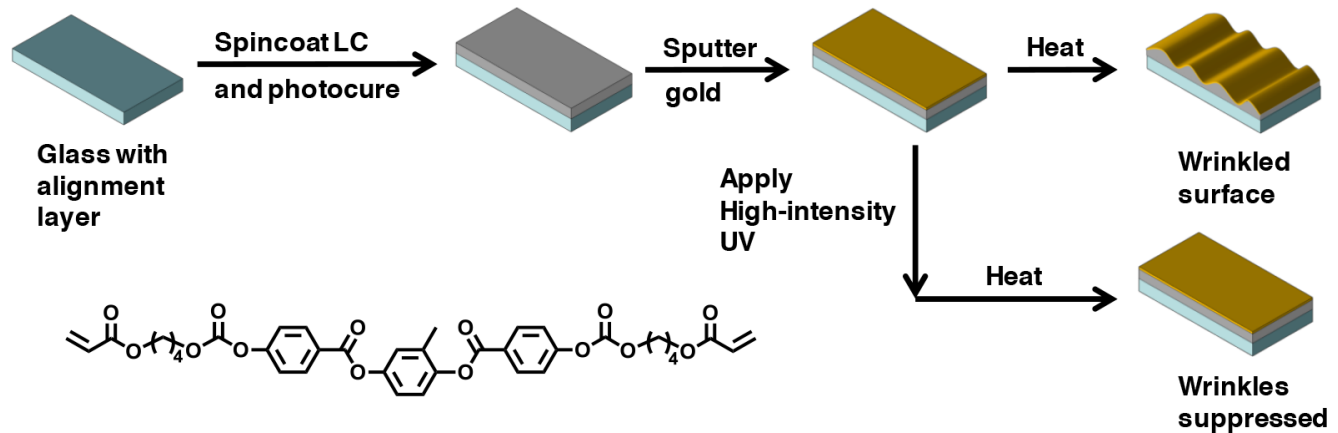

Figure 1. Schematic overview of the procedure of wrinkle generation and suppression, including the reactive mesogen which was used to prepare the polymer film.
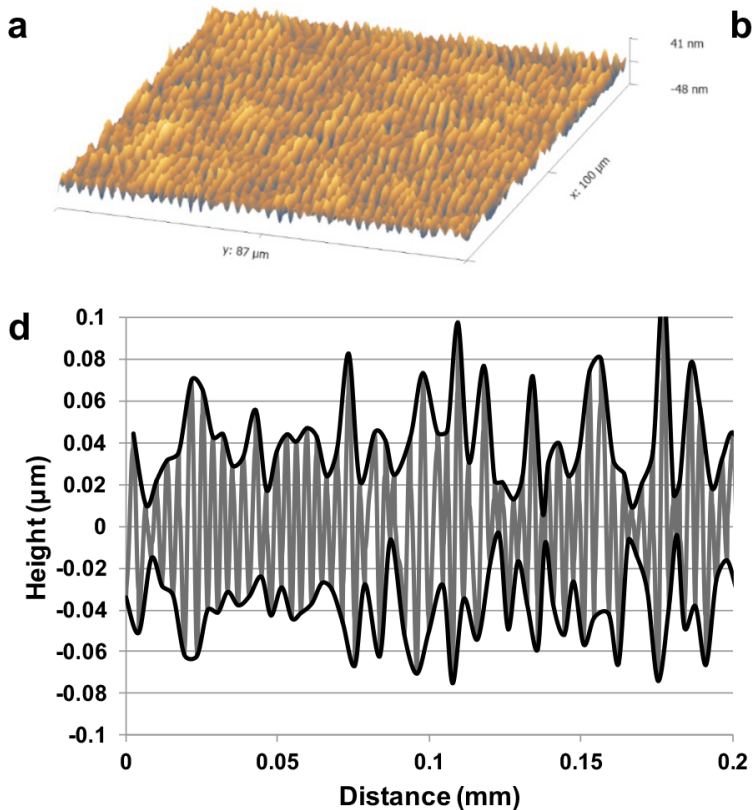

b
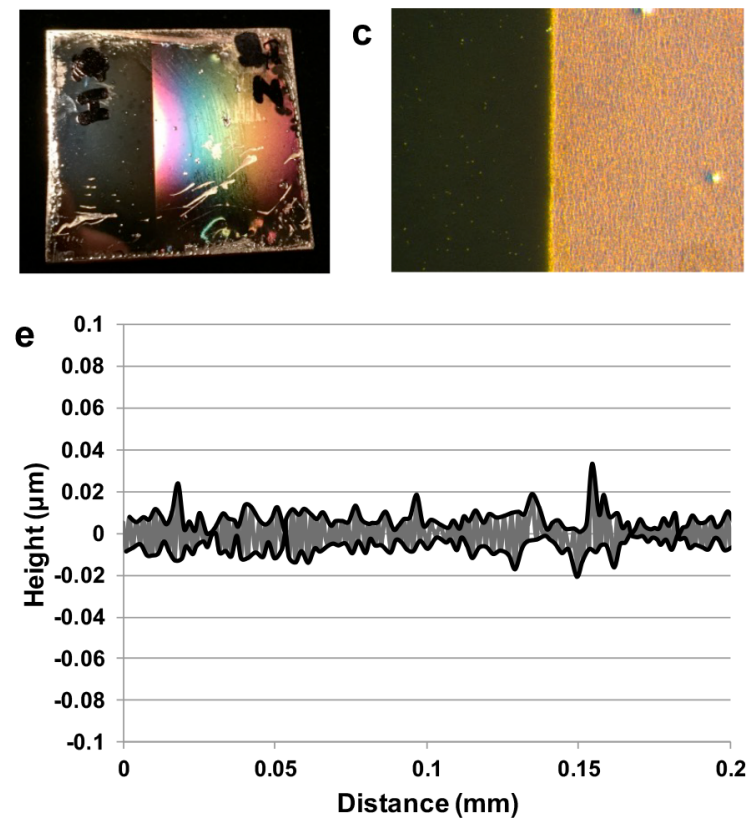

Figure 2. (a) Example of a wrinkled LC/gold film, as observed using surface profilometry. (b) Photograph of a gold-coated LC sample after wrinkling. The left side was exposed to $18 \mathrm{~J} / \mathrm{cm}^{2} \mathrm{UV}$ light at $28.9 \mathrm{~mW} / \mathrm{cm}^{2}$ after the gold layer was applied. A clear difference in reflective properties can be observed. (c) An $\sim 30 \times$ optical microscope image of the boundary between an area exposed to UV light (left side of image) and a nonexposed area (right side of the image) of the same sample displayed in panel b. (d) Profile section of an LC bilayer/gold after wrinkling. The profile is plotted in gray, while the minima and maxima are plotted in black. (e) Similar profile section of the same bilayer film as shown in panel d, to which a $10.6 \mathrm{~J} / \mathrm{cm}^{2}$ dose of UVA light was applied before heating. The wrinkling amplitude and wavelength are visibly diminished.

through UV irradiation using a relatively weak facial UV lamp $\left(0.88 \mathrm{~mW} / \mathrm{cm}^{2}\right)$ for $15-20 \mathrm{~min}$. Subsequently, a $\sim 20 \mathrm{~nm}$ gold layer was applied to the polymer film via sputter coating. Wrinkling could be induced in the bilayer systems by heating them beyond the glass transition temperature of the polymer. The wrinkles always followed the alignment direction of the mesogenic units, as was described previously. ${ }^{44}$ The procedure is outlined in Figure 1. A surface profilometer was used to visualize the wrinkles. An example of this is shown in Figure 2a.

To study the effect of a high-intensity UV dose on the bilayer, the combined films were exposed to a higher intensity light source $\left(9-21 \mathrm{~mW} / \mathrm{cm}^{2}\right.$ UVA light) through the glass backing layer, with a chromium mask partially shading the film from light exposure. The resulting samples were subsequently heated beyond the glass transition to induce wrinkling. In general, visual inspection revealed an obvious diminishing of the wrinkle formation in the exposed areas, as illustrated by an example shown in Figure 2, parts b and c. The 2D profiles of the films were measured on both the exposed and nonexposed regions. An example of this is shown in Figure 2, parts $\mathrm{d}$ and e. From these measurements it became clear that both the average wrinkle amplitude and wavelength were diminished on the irradiated side compared to the nonirradiated side.

This effect, in combination with photoalignment of the mesogenic monomers, opens up the opportunity to form remarkably complex wrinkling patterns in the bilayers through a straightforward procedure, using multiple selective UV exposures. An example of this is shown in Figure 3a, using three mask exposures that together form an asymmetric image of two elephants. First, a photosensitive alignment material was spin-coated onto a glass slide. Two mask exposures were carried out to induce different alignment directions in two different areas of the alignment layer. Then, the monomer mixture was applied, which aligned with the alignment layers. The monomer was polymerized using low-intensity ultraviolet light, and the gold layer was applied. A third exposure with high-intensity UV light was carried out to generate a background area with suppressed wrinkling, outside of the 
a

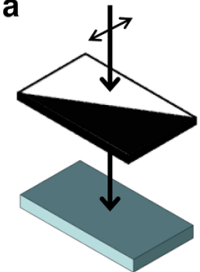

UV exposure 1

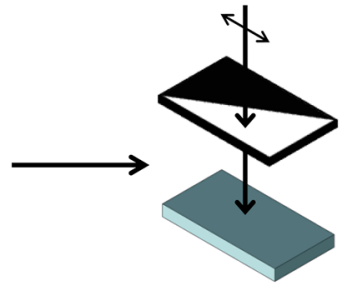

UV exposure 2

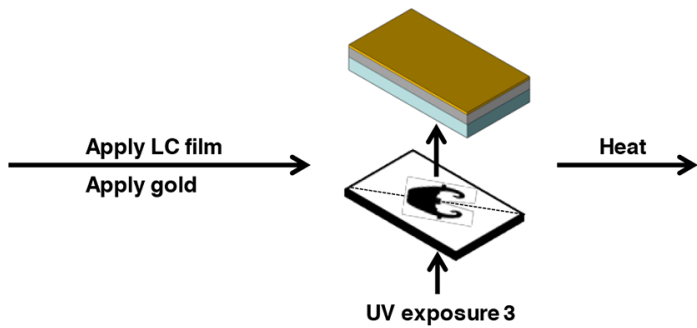

b
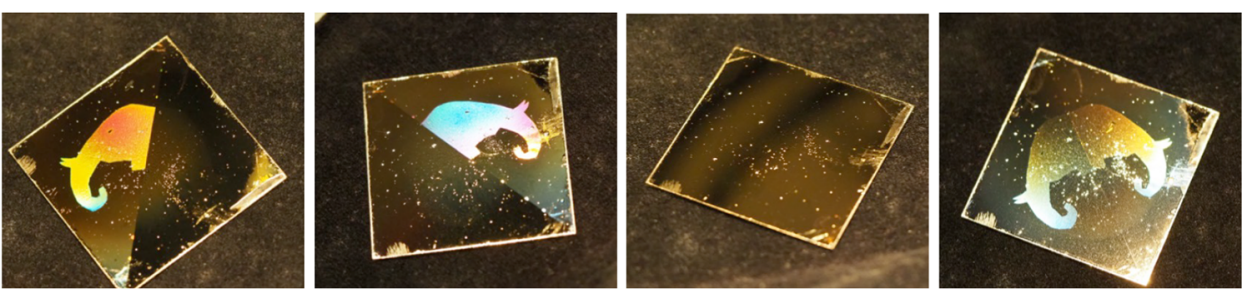

Figure 3. (a) Procedure to prepare a wrinkled LC/gold surface with an asymmetric image of two elephants. Two photoalignment steps (ref 44) to align the photoalignment layers were followed by spin coating of the LC, polymerization, and sputtering of the gold layer. Then, a third exposure to high-intensity UV light through a third mask to generate the image of the elephants was carried out. (b) Photographs of the patterned surface from different angles. Depending on the viewing angle, the left or the right elephant was visible, neither of them were visible, or both were visible. The color of the image was also angular-dependent. The film was $5 \times 5 \mathrm{~cm}^{2}$ in size.
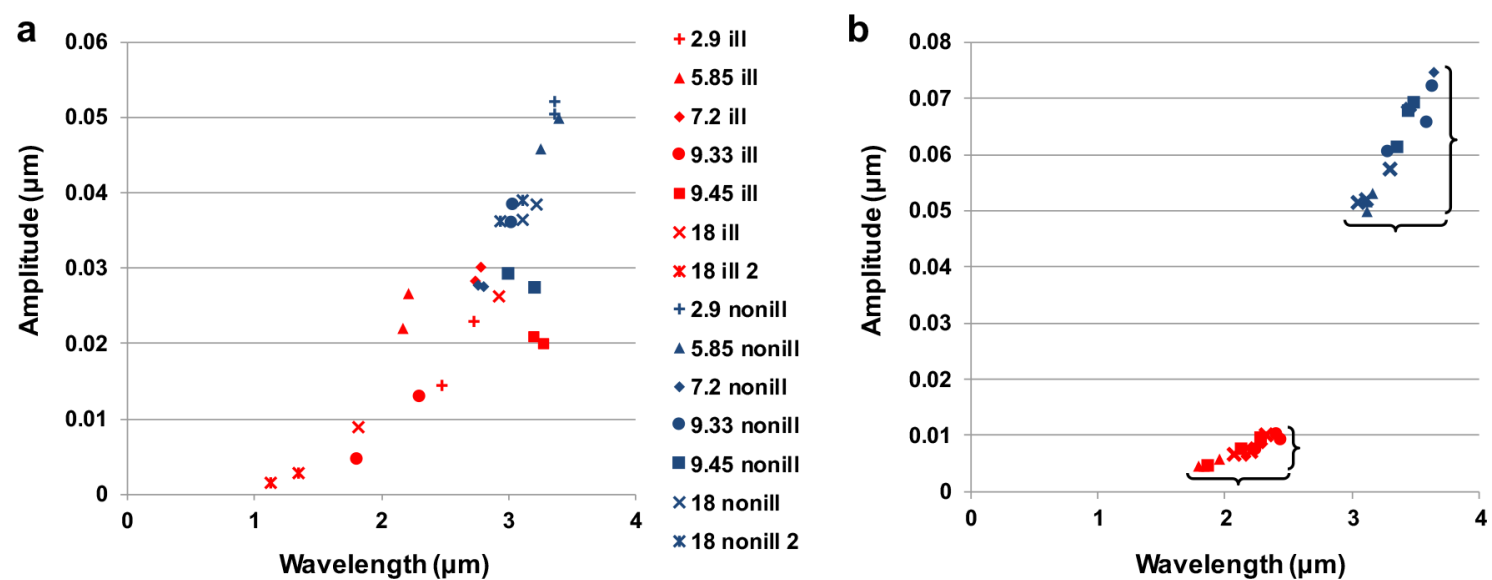

Figure 4. (a) Average amplitude and wavelength of wrinkled bilayer systems which were exposed to various intensities of UVA light. Each symbol indicates a different total dose (there were two films which were exposed to $18 \mathrm{~J} / \mathrm{cm}^{2}$ ). Red symbols represent data measured in irradiated areas, while blue symbols represent data measured in nonirradiated areas. The numbers in the legend indicate the total dose (in J/ $\left.\mathrm{cm}^{2}\right)$. Each film was measured twice on each side. (b) Average amplitude and wavelength of wrinkled bilayer systems which were all exposed to the same UV dose at the same intensity $\left(20.6 \mathrm{~mW} / \mathrm{cm}^{2}, 6.18 \mathrm{~J} / \mathrm{cm}^{2}\right)$. There were five films, and each film was measured three times on each side.

image of the two elephants. Heating beyond the glass transition revealed the final image, as can be seen in Figure 3b: the unexposed areas have aligned wrinkles and act as diffraction gratings which are positioned in different directions, while the background has unaligned wrinkles and appears glossy. As such, the image of the elephants is sharply visible, with the asymmetry becoming obvious when the surface is viewed from different angles: either one of the elephants is visible, none of them is visible, or both are visible, depending on the viewing angle. The color of the elephants is also angledependent. This image could not have been created using only photoalignment, as the background would also diffract light which would interfere with the image. These results shows that our new procedure is more versatile and more convenient compared to our previously reported photoalignment results, allowing localized control over wrinkle dimensions and direction through simple photomasking procedures.
To better understand the mechanism of the suppression, the effect of the UV dose on the wrinkling amplitude and period was studied. To this end, several samples were prepared and partially irradiated at various UV light intensities. After wrinkling, the $2 \mathrm{D}$ profile was measured twice on each of the irradiated and nonirradiated sides. The average amplitude and period were then calculated from each measurement. The results are plotted in Figure 4a. From the plot, it became clear that, in general, both the average amplitude and wavelength were reduced upon UV exposure. However, the relationship between dose and effect seemed to be somewhat inconsistent. Also, there was a rather large spread in wavelength and amplitude between the samples on the side where no UV dose was applied, which is probably caused by slight variations in gold layer thickness or polymer modulus. On the other hand, there seemed to be a positive relation between the wavelength and amplitude both before and after UV exposure. A second series of samples was prepared, which were all partially 
irradiated with the same UV dose $\left(20.6 \mathrm{~mW} / \mathrm{cm}^{2}\right.$ for $300 \mathrm{~s}$, $6.18 \mathrm{~J} / \mathrm{cm}^{2}$ ), the results of which can be found in Figure $4 \mathrm{~b}$. Again, a spread in amplitude and wavelength was found on both the nonirradiated and irradiated sides and a reduced amplitude and wavelength on the exposed side. It was also found that the spread in wavelength is the same on both sides, while the spread in amplitude is strongly reduced on the exposed side.

It would be reasonable to assume the change in amplitude and wavelength upon exposure to the high-intensity light is caused by an increase in stiffness of the LC polymer layer due to increased cross-linking. ${ }^{46,47}$ FT-IR was measured to determine the degree of polymerization of the samples after initial low-intensity UV exposure during polymerization. The IR spectra of pure LC-242, polymerized LC-242, and polymerized LC-242 after high-intensity UV light exposure (seen in Figure S1) suggest that polymerization has essentially completed before the high-intensity dose was applied, which suggests that it is not increased cross-linking of the LC polymer which is responsible for the suppression effect. Measurements of the bulk modulus were done by DMTA both after polymerization and after exposure to the highintensity lamp for $10 \mathrm{~min}$ (dose $9.2-10.5 \mathrm{~J} / \mathrm{cm}^{2}$ ); no significant difference in the modulus or glass transition temperatures could be determined (see Figure S2), so if the mechanism involves a modulus increase of the polymer, it only happens at the polymer-gold interface.

From the ImAFM data (see Figure S3), the average values of the modulus for the region near the surface can be extracted and are summarized in the Table S1. It appears that, as expected, the surface modulus of the nonexposed areas is not modified, but the surface modulus of the exposed areas increases with exposure dose, ranging from $0.6 \mathrm{GPa}(1.9 \mathrm{~J} /$ $\left.\mathrm{cm}^{2}\right)$ to $2.7 \mathrm{GPa}$ for the larger dose $\left(18 \mathrm{~J} / \mathrm{cm}^{2}\right)$.

The resistivity of the gold layer in both the exposed and unexposed areas was determined to be the same, and scanning electron microscopy (SEM) images (see Figure S4 in the Supporting Information) of the film surface showed no significant cracks or additional features in the exposed areas to suggest a weakening of the gold layer. An experiment was carried out to determine if the presence of the gold layer during high-intensity UV light exposure was necessary for the suppression effect to take place. This was done by exposing half of a sample to $11.75 \mathrm{~J} / \mathrm{cm}^{2} \mathrm{UV}$ before sputtering with gold. There was only a minor difference between the regions exposed to UV before gold sputtering and those unexposed to UV light before sputtering, suggesting the presence of gold is necessary for the wrinkle suppression to take place upon highintensity UV exposure. The experiment was repeated once with the same result.

A wrinkling system can be described by two formulas: $\lambda=$ $\pi h\left(E_{\mathrm{m}} / 3 E_{\mathrm{p}}\right)^{1 / 3}$, where $\lambda$ is the wrinkling period, $h$ is the thickness of the metal film, $E_{\mathrm{m}}$ is the modulus of the metal film, and $E_{\mathrm{p}}$ is the modulus of the polymer, and $\delta=A^{2} / \lambda^{2}$, where $\delta$ is the stress and $A$ is the wrinkling amplitude. It can be assumed that $h$ and $E_{\mathrm{m}}$ are not affected by the UV irradiation, so it seems that the suppression effect is caused either by an increase in $E_{\mathrm{p}}$ or a decrease in $\delta$. As the only variables that affect $\lambda$ are $h, E_{\mathrm{m}}$, and $E_{\mathrm{p}}$, it must be concluded that $E_{\mathrm{p}}$ goes up. As stated before, bulk measurements of UV-exposed materials do not show this, and UV exposure only causes wrinkle suppression after the gold is already in place, so it has to be an effect that only takes place on the interface between the metal and the polymer. The surface ImAFM measurements indicated increased surface modulus in the regions exposed to highintensity UVA light (see Figure S3), supporting the supposition that the stiffening effect is confined to close to the gold/LC interface. A factor 4 in modulus is observed between the nonexposed areas and the area exposed to $18 \mathrm{~J} /$ $\mathrm{cm}^{2}$. In addition, as there is a linear relationship between $\lambda$ and $A$, the distribution of these values should be preserved after irradiation, which is not what is observed, meaning that $\delta$ must change as well. As the stress is induced during sputter coating, this means that dissipation of the stress also plays a part in UVinduced wrinkle suppression. Combined, the data suggest that the active mechanism involves photodegradation of the polymer layer at the polymer-gold interface, possibly due to the formation of mechanoradicals. It is known from other studies that stressed polymers degrade faster as a result of the appearance of sub-micrometer cracks. ${ }^{48-50}$ However, more research is needed to confirm whether this is the case here.

\section{CONCLUSIONS}

We have shown that high-intensity UV irradiation can be used to decrease the average amplitude and wavelength of the surface wrinkles in an LC polymer/gold bilayer system. As the wrinkle direction is locally controlled by the alignment of the molecules, and the wavelength and amplitude are locally controlled by the second high-intensity UV exposure, it is now possible to influence all relevant characteristics of the formed surface structures. This expands the range of patterned surfaces that can be prepared, allows for the preparation of remarkably complex wrinkle patterns, requires no complicated and expensive printing or lithography techniques, and increases the number of potential applications for this system. Measurements of average wavelength and amplitude of the wrinkles with and without UV exposure, combined with bulk modulus measurements and surface modulus measurements, indicate that the mechanism involves an accelerated photodegradation process in the polymer at the polymer-gold interface under stress, potentially due to the formation of mechanoradicals. This mechanism needs to be further studied to improve the fine-tuning of the wrinkle dimensions with light.

\section{ASSOCIATED CONTENT}

\section{S Supporting Information}

The Supporting Information is available free of charge on the ACS Publications website at DOI: 10.1021/acs.langmuir.8b01934.

IR spectra of nonpolymerized and polymerized LC samples, storage modulus of representative LC films, DMT modulus mapping of non-UV-illuminated and UV-illuminated films, SEM image of illuminated and nonilluminated regions of LC film, and a table of measured surface moduli of variously UV-exposed LC films (PDF)

\section{AUTHOR INFORMATION}

\section{Corresponding Author}

*E-mail: m.g.debije@tue.nl.

ORCID

Laurens T. de Haan: 0000-0003-4978-1808

Guofu Zhou: 0000-0003-1101-1947 
Michael G. Debije: 0000-0001-8844-1115

Notes

The authors declare no competing financial interest.

\section{ACKNOWLEDGMENTS}

This work was financially supported by the National Natural Science Foundation of China (Nos. 51561135014 and U1501244), the Guangdong Innovative Research Team Program (No. 2013C102), the Major Science and Technology Projects of Guangdong Province (No. 2017B020240002), the Shenzhen Science and Technology Plan (No. JSGG20170414143009027), the Guangdong Provincial Key Laboratory of Optical Information Materials and Technology (No. 2017B030301007), the MOE International Laboratory for Optical Information Technologies, and the 111 Project. P.L. is a Senior Research Associate of FRS-FNRS (Belgium). The authors would like to thank Nathan Otermans for his assistance with some of the sample preparations, Jitesh Barman for helpful discussions, and Dario Cambie for his careful reading of the text.

\section{REFERENCES}

(1) Bae, W.-G.; Kim, H. N.; Kim, D.; Park, S.-H.; Jeong, H. E.; Suh, K.-Y. Scalable Multiscale Patterned Structures Inspired by Nature: The Role of Hierarchy. Adv. Mater. 2014, 26, 675-700.

(2) Huang, J.; Wang, X.; Wang, Z. L. Controlled Replication of Butterfly Wings for Achieving Tunable Photonic Properties. Nano Lett. 2006, 6, 2325-2331.

(3) Li, X.-M.; Reinhoudt, D.; Crego-Calama, M. What Do We Need for a Superhydrophobic Surface? A Review on the Recent Progress in the Preparation of Superhydrophobic Surfaces. Chem. Soc. Rev. 2007, $36,1350$.

(4) Xue, C.; Xiang, J.; Nemati, H.; Bisoyi, H. K.; Gutierrez-Cuevas, K.; Wang, L.; Gao, M.; Zhou, S.; Yang, D.; Lavrentovich, O. D. LightDriven Reversible Alignment Switching of Liquid Crystals Enabled by Azo Thiol Grafted Gold Nanoparticles. ChemPhysChem 2015, 16, $1852-1856$.

(5) Bisoyi, H. K.; Li, Q. Light-Driven Liquid Crystalline Materials: From Photo-Induced Phase Transitions and Property Modulations to Applications. Chem. Rev. 2016, 116, 15089-15166.

(6) Schuh, C.; Lomadze, N.; Rühe, J.; Kopyshev, A.; Santer, S. Photomechanical Degrafting of Azo-Functionalized Poly(Methacrylic Acid) (PMAA) Brushes. J. Phys. Chem. B 2011, 115, 10431-10438.

(7) Chen, C.-M.; Yang, S. Wrinkling Instabilities in Polymer Films and Their Applications. Polym. Int. 2012, 61, 1041-1047.

(8) Chan, E. P.; Crosby, A. J. Fabricating Microlens Arrays by Surface Wrinkling. Adv. Mater. 2006, 18, 3238-3242.

(9) Efimenko, K.; Finlay, J.; Callow, M. E.; Callow, J. A.; Genzer, J. Development and Testing of Hierarchically Wrinkled Coatings for Marine Antifouling. ACS Appl. Mater. Interfaces 2009, 1, 1031-1040.

(10) Greco, F.; Fujie, T.; Ricotti, L.; Taccola, S.; Mazzolai, B.; Mattoli, V. Microwrinkled Conducting Polymer Interface for Anisotropic Multicellular Alignment. ACS Appl. Mater. Interfaces 2013, 5, 573-584.

(11) Yang, P.; Baker, R. M.; Henderson, J. H.; Mather, P. T. In Vitro Wrinkle Formation via Shape Memory Dynamically Aligns Adherent Cells. Soft Matter 2013, 9, 4705-4714.

(12) Harrison, C.; Stafford, C. M.; Zhang, W.; Karim, A. Sinusoidal Phase Grating Created by a Tunably Buckled Surface. Appl. Phys. Lett. 2004, 85, 4016-4018.

(13) Li, Y.; Dai, S.; John, J.; Carter, K. R. Superhydrophobic Surfaces from Hierarchically Structured Wrinkled Polymers. ACS Appl. Mater. Interfaces 2013, 5, 11066-11073.

(14) Genzer, J.; Groenewold, J. Soft Matter with Hard Skin: From Skin Wrinkles to Templating and Material Characterization. Soft Matter 2006, 2, 310.
(15) Agrawal, A.; Luchette, P.; Palffy-Muhoray, P.; Biswal, S. L.; Chapman, W. G.; Verduzco, R. Surface Wrinkling in Liquid Crystal Elastomers. Soft Matter 2012, 8, 7138.

(16) Agrawal, A.; Yun, T.; Pesek, S. L.; Chapman, W. G.; Verduzco, R. Shape-Responsive Liquid Crystal Elastomer Bilayers. Soft Matter 2014, 10, 1411-1415.

(17) Chen, Y.-C.; Crosby, A. J. Wrinkling of Inhomogeneously Strained Thin Polymer Films. Soft Matter 2013, 9, 43-47.

(18) Davis, C. S.; Crosby, A. J. Wrinkle Morphologies with Two Distinct Wavelengths. J. Polym. Sci., Part B: Polym. Phys. 2012, 50, $1225-1232$

(19) Ebata, Y.; Croll, A. B.; Crosby, A. J. Wrinkling and Strain Localizations in Polymer Thin Films. Soft Matter 2012, 8, 9086.

(20) Bowden, N.; Brittain, S.; Evans, A. A. G.; Hutchinson, J. W.; Whitesides, G. M. Spontaneous Formation of Ordered Structures in Thin Films of Metals Supported on an Elastomeric Polymer. Nature 1998, 393, 146-149.

(21) Casper, M. D.; Gözen, A. Ö.; Dickey, M. D.; Genzer, J.; Maria, J.-P. Surface Wrinkling by Chemical Modification of Poly(Dimethylsiloxane)-Based Networks during Sputtering. Soft Matter 2013, 9, 7797.

(22) Wu, D.; Yin, Y.; Xie, H.; Shang, Y.; Li, C.; Wu, L.; Dai, X. Controlling the Surface Buckling Wrinkles by Patterning the Material System of Hard-Nano-Film/Soft-Matter-Substrate. Sci. China: Phys., Mech. Astron. 2014, 57, 637-643.

(23) Huck, W. T. S.; Bowden, N.; Onck, P.; Pardoen, T.; Hutchinson, J. W.; Whitesides, G. M. Ordering of Spontaneously Formed Buckles on Planar Surfaces. Langmuir 2000, 16, 3497-3501.

(24) Ahn, S. H.; Guo, L. J. Spontaneous Formation of Periodic Nanostructures by Localized Dynamic Wrinkling. Nano Lett. 2010, $10,4228-4234$.

(25) Guo, C. F.; Nayyar, V.; Zhang, Z.; Chen, Y.; Miao, J.; Huang, R.; Liu, Q. Path-Guided Wrinkling of Nanoscale Metal Films. Adv. Mater. 2012, 24, 3010-3014.

(26) Okayasu, T.; Zhang, H. L.; Bucknall, D. G.; Briggs, G. A. D. Spontaneous Formation of Ordered Lateral Patterns in Polymer ThinFilm Structures. Adv. Funct. Mater. 2004, 14, 1081-1088.

(27) Cao, C.; Chan, H. F.; Zang, J.; Leong, K. W.; Zhao, X. Harnessing Localized Ridges for High-Aspect-Ratio Hierarchical Patterns with Dynamic Tunability and Multifunctionality. Adv. Mater. 2014, 26, 1763-1770.

(28) Chen, Z.; Young Kim, Y.; Krishnaswamy, S. Anisotropic Wrinkle Formation on Shape Memory Polymer Substrates. J. Appl. Phys. 2012, 112, 124319.

(29) Bowden, N.; Huck, W. T. S.; Paul, K. E.; Whitesides, G. M. The Controlled Formation of Ordered, Sinusoidal Structures by Plasma Oxidation of an Elastomeric Polymer. Appl. Phys. Lett. 1999, 75, 2557-2559.

(30) Breid, D.; Crosby, A. J. Effect of Stress State on Wrinkle Morphology. Soft Matter 2011, 7, 4490.

(31) Ding, W.; Yang, Y.; Zhao, Y.; Jiang, S.; Cao, Y.; Lu, C. WellDefined Orthogonal Surface Wrinkles Directed by the Wrinkled Boundary. Soft Matter 2013, 9, 3720.

(32) Kang, S. H.; Na, J.-H.; Moon, S. N.; Lee, W. I.; Yoo, P. J.; Lee, S.-D. Self-Organized Anisotropic Wrinkling of Molecularly Aligned Liquid Crystalline Polymer. Langmuir 2012, 28, 3576-3582.

(33) Chen, C.-M.; Reed, J. C.; Yang, S. Guided Wrinkling in Swollen, Pre-Patterned Photoresist Thin Films with a Crosslinking Gradient. Soft Matter 2013, 9, 11007.

(34) Chung, J. Y.; Nolte, A. J.; Stafford, C. M. Diffusion-Controlled, Self-Organized Growth of Symmetric Wrinkling Patterns. Adv. Mater. 2009, 21, 1358-1362.

(35) Efimenko, K.; Rackaitis, M.; Manias, E.; Vaziri, A.; Mahadevan, L.; Genzer, J. Nested Self-Similar Wrinkling Patterns in Skins. Nat. Mater. 2005, 4, 293-297.

(36) Ohzono, T.; Watanabe, H.; Vendamme, R.; Kamaga, C.; Kunitake, T.; Ishihara, T.; Shimomura, M. Spatial Forcing of SelfOrganized Microwrinkles by Periodic Nanopatterns. Adv. Mater. 2007, 19, 3229-3232. 
(37) Vandeparre, H.; Léopoldès, J.; Poulard, C.; Desprez, S.; Derue, G.; Gay, C.; Damman, P. Slippery or Sticky Boundary Conditions: Control of Wrinkling in Metal-Capped Thin Polymer Films by Selective Adhesion to Substrates. Phys. Rev. Lett. 2007, 99, 188302.

(38) Yoo, P. J.; Suh, K. Y.; Park, S. Y.; Lee, H. H. Physical SelfAssembly of Microstructures by Anisotropic Buckling. Adv. Mater. 2002, 14, 1383-1387.

(39) Khang, D.-Y. A Stretchable Form of Single-Crystal Silicon for High-Performance Electronics on Rubber Substrates. Science 2006, $311,208-212$.

(40) Hendricks, T. R.; Lee, I. Wrinkle-Free Nanomechanical Film: Control and Prevention of Polymer Film Buckling. Nano Lett. 2007, 7, 372-379.

(41) Thornton, J. A.; Hoffman, D. W. Stress-Related Effects in Thin Films. Thin Solid Films 1989, 171, 5-31.

(42) Detor, A. J.; Hodge, A. M.; Chason, E.; Wang, Y.; Xu, H.; Conyers, M.; Nikroo, A.; Hamza, A. Stress and Microstructure Evolution in Thick Sputtered Films. Acta Mater. 2009, 57, 20552065.

(43) Vandeparre, H.; Damman, P. Wrinkling of Stimuloresponsive Surfaces: Mechanical Instability Coupled to Diffusion. Phys. Rev. Lett. 2008, 101, 124301.

(44) De Haan, L. T.; Leclère, P.; Damman, P.; Schenning, A. P. H. J.; Debije, M. G. On-Demand Wrinkling Patterns in Thin Metal Films Generated from Self-Assembling Liquid Crystals. Adv. Funct. Mater. 2015, 25, 1360-1365.

(45) Platz, D.; Tholén, E. A.; Pesen, D.; Haviland, D. B. Intermodulation Atomic Force Microscopy. Appl. Phys. Lett. 2008, 92, 153106 .

(46) Kakani, S. M.; Zadgaonkar, S. S.; Pande, S. A.; Pande, K. N.; Peshwe, D. R. Study and Evaluation on Effect of Ultra-Violet (UV) Radiation on the Structural and Mechanical Properties of Talc Filled Polypropylene Co-Polymer (TFPP). Trans. Indian Inst. Met. 2013, 66, 273-280.

(47) Warner, W. C.; Gruber, E. E. Light Aging of a Polyblend Film under Interference Filters. Ind. Eng. Chem. Prod. Res. Dev. 1966, 5, 219-221.

(48) Rabek, J. F. Polymer Photodegradation: Mechanisms and Experimental Methods; Chapman \& Hall: London, 1995.

(49) O’Donnell, B.; White, J. R. Stress-Accelerated Photo-Oxidation of Polypropylene and Glass-Fibre-Reinforced Polypropylene. Polym. Degrad. Stab. 1994, 44, 211-222.

(50) Baumhardt-Neto, R.; De Paoli, M.-A. Mechanical Degradation of Polypropylene: Effect of UV Irradiation. Polym. Degrad. Stab. 1993, $40,59-64$. 